\title{
BMJ Open Are COVID-19 models blind to the social determinants of health? A systematic review protocol
}

\author{
Ava John-Baptiste, ${ }^{1,2,3,4}$ Marc S Moulin (D) , ${ }^{1}$ Shehzad Ali ${ }^{1,2,3,5}$
}

\begin{abstract}
To cite: John-Baptiste A, Moulin MS, Ali S. Are COVID-19 models blind to the social determinants of health? A systematic review protocol. BMJ Open 2021;11:e048995. doi:10.1136/ bmjopen-2021-048995

- Prepublication history for this paper is available online. To view these files, please visit the journal online (http://dx.doi. org/10.1136/bmjopen-2021 048995).
\end{abstract}

Received 12 January 2021 Accepted 18 May 2021

Check for updates

(C) Author(s) (or their employer(s)) 2021. Re-use permitted under CC BY-NC. No commercial re-use. See rights and permissions. Published by BMJ.

${ }^{1}$ Department of Anesthesia and Perioperative Medicine, Western University, London, Ontario,

Canada

${ }^{2}$ Department of Epidemiology and Biostatistics, Western University, London, Ontario, Canada

${ }^{3}$ Interfaculty Program in Public Health, Western University, London, Ontario, Canada

${ }^{4}$ Lawson Health Research Institute, London, Ontario, Canada

${ }^{5}$ WHO Collaborating Centre for Knowledge Translation and Health Technology Assessment in Health Equity, Ottawa, Ontario, Canada

Correspondence to

Dr Ava John-Baptiste;

ajohnbap@uwo.ca

\section{ABSTRACT}

Introduction Infectious disease models are important tools to inform public health policy decisions. These models are primarily based on an average population approach and often ignore the role of social determinants in predicting the course of a pandemic and the impact of policy interventions. Ignoring social determinants in models may cause or exacerbate inequalities. This limitation has not been previously explored in the context of the current pandemic, where COVID-19 has been found to disproportionately affect marginalised racial, ethnic and socioeconomic groups. Therefore, our primary goal is to identify the extent to which COVID-19 models incorporate the social determinants of health in predicting outcomes of the pandemic.

Methods and analysis We will search MEDLINE, EMBASE, Cochrane Library and Web of Science databases from December 2019 to August 2020. We will assess all infectious disease modelling studies for inclusion of social factors that meet the following criteria: (a) focused on human spread of SARS-CoV-2; (b) modelling studies; (c) interventional or non-interventional studies; and (d) focused on one of the following outcomes: COVID-19related outcomes (eg, cases, deaths), non-COVID-19related outcomes (ie, impacts of the pandemic or control policies on other health conditions or health services), or impact of the pandemic or control policies on economic outcomes. Data will only be extracted from models incorporating social factors. We will report the percentage of models that considered social factors, indicate which social factors were considered, and describe how social factors were incorporated into the conceptualisation and implementation of the infectious disease models. The extracted data will also be used to create a narrative synthesis of the results.

Ethics and dissemination Ethics approval is not required as only secondary data will be collected. The results of this systematic review will be disseminated through peerreviewed publication and conference proceedings. PROSPERO registration number CRD42020207706.

\section{INTRODUCTION}

In the early months of the pandemic, COVID-19 was described by New York Governor Andrew Cuomo as the 'great equaliser' ${ }^{\text {' }}$ as the new coronavirus swept through society without bias. A shared lack of immunity meant that regardless of prestige, wealth,

\section{Strengths and limitations of this study}

The Preferred Reporting Items for Systematic Review and Meta-Analysis Protocols will be followed.

- A systematic approach to evaluating modellers' incorporation of social factors into COVID-19 models will identify practical approaches to better quantify social inequities of the pandemic and predict the impact of public policies on marginalised populations.

- Included studies of COVID-19 models may not be amenable to quantitative synthesis due to heterogeneity of the literature.

- Given the time frame of the search strategy and the rapid evolution of COVID-19 modelling literature, the results may not include studies evaluating certain prevention strategies, such as vaccination.

fame or age, all were thought to be equally at risk. ${ }^{1}$ However, epidemiological researchers quickly demonstrated that this was, and remains to be, far from the truth. The pandemic has put existing health inequalities under the microscope and highlighted that COVID-19 has disproportionately affected marginalised racial, ethnic, and socioeconomic groups through its unequal health burden and its disparity of economic losses. ${ }^{2} 3$ A recent investigation found that among 158 counties in large US metropolitan areas, those with predominantly non-white populations had infection rates approximately eight times higher, and a fatality rate more than nine times higher than counties with a mostly white population. ${ }^{4}$ Similarly, people living in the poorest neighbourhoods of England and Wales have been found to be twice as likely to die from COVID-19 as those in more affluent areas. ${ }^{5}$ Examples of similar trends can be found throughout history. Perhaps most notably in terms of size and scale, data collected from the 1918 influenza pandemic demonstrated that non-white minorities had higher all-cause mortality and influenzarelated mortality rates when compared with their white counterparts. ${ }^{6}$ These disparities are a symptom of deeper societal and health 
system inequities, including disproportionate exposure through high-risk jobs, prevalence of comorbidities, and inequitable access to testing and treatment.

Over the past century, infectious disease models have played a fundamental role in helping public health policymakers implement infection control interventions, as well as shape the theories used in disease mitigation. ${ }^{7}$ Infectious disease models have been described as, 'a scientific approach to formulating an explanation for an observed phenomenon and then testing this formulation to project the outcome of various experiments under pertinent conditions ${ }^{, 8}(\mathrm{p} 1)$. Models provide a framework for experts to study a number of important factors related to the SARS-CoV-2 pandemic, including the following:

1. Viral transmission dynamics-models use observed data such as the number of confirmed cases, to estimate difficult-to-observe transmission dynamics, such as the incubation period and percentage of asymptomatic spread in the population. ${ }^{9} 10$

2. Anticipate the future course of an outbreak-models forecast future incidence, prevalence, morbidity and mortality, based on input parameters. ${ }^{112}$

3. Guide public health planning and infectious disease control-models help researchers simulate real-world possibilities and thus appraise the potential impact of disease-control strategies such as mask wearing, school closures, testing and contract-tracing on the population. ${ }^{1314}$

4. Models can estimate a range of outcomes, those directly related to COVID-19 or secondary effects of the disease and control measures, on population health, health service utilisation, and economic outcomes such as cost and quality of life. ${ }^{15}$

Infectious disease models can only be fit for purpose if the models are appropriately conceptualised and model parameters are based on the best available evidence. ${ }^{16}{ }^{17}$ Model conceptualisation requires decisions about the model structure, including which health states to include, division of the population into subgroups and selection of characteristics to define the subgroups. ${ }^{16}$ Thus, models can incorporate the social determinants of health, ${ }^{18}$ such as income, education, employment and ethnicity. However, structuring models to reflect social determinants increases complexity and sometimes, due to limited availability of data, raises questions about structural uncertainty. ${ }^{19}{ }^{20}$ Complexity and uncertainty may lead modellers to simplify and favour average population models foregoing appraisal of different types of individuals or important subgroups. ${ }^{19-21}$ Current evidence highlights the importance of social factors in predicting the course of the pandemic and in analyses of policy options, ${ }^{2-6}$ although it is unclear to what extent current infectious disease models are based on an average population approach and ignore social factors. To the best of our knowledge, this limitation has yet to be explored in the context of the COVID-19 pandemic. Therefore, the present systematic review will aim to answer the following research question: 'To what extent do COVID-19 models incorporate the social determinants of health?' Our objective is to systematically review human COVID-19 modelling studies (predictive, forecasting) to identify what proportion of models incorporate social factors and for included modelling studies, to identify how social factors were incorporated into the conceptualisation of the infectious disease models. The work will provide an important reflection on COVID-19 modelling practices and can lead to development of a blueprint for other modellers to incorporate social factors in the future. We hope that this review will lead to infectious disease models that explicitly acknowledge the role of social determinants in making better predictions as well as mitigating inequalities, and therefore result in better infectious disease outcomes.

\section{METHODS}

\section{Protocol and registration}

This systematic review will be conducted according to the Preferred Reporting Items for Systematic Reviews and Meta-analyses guidelines ${ }^{22}$ for the identification, screening, eligibility and inclusion of retrieved research studies. The current protocol is registered in the International Prospective Register of Systematic Reviews. ${ }^{23}$ The anticipated start date for the proposed systematic review is November 2020. The full results will be submitted for peer-reviewed publication immediately following completion of quality assessment and the drafting of a manuscript in August 2021.

\section{Search strategy and eligibility criteria}

MEDLINE, EMBASE, Cochrane Library and Web of Science databases will be searched. The present systematic review is designed to have broad eligibility criteria to identify all COVID-19 modelling studies published from December 2019 to 14 August 2020. Studies will be initially eligible if they: (1) have a human SARS-CoV-2 population; (2) are a modelling study (eg, mechanistic, mathematical, network, simulation, auto-regressive, Markov or other model); and (3) investigate one of the following outcomes: COVID-19 disease-related outcomes (eg, cases, hospitalisations, deaths, recoveries), non-COVID-19 disease-related outcomes (eg, impact on other health conditions such as mental health), impacts on health services (eg, bed occupancy, ventilators, surgical delays), impact of policies (eg, lockdowns, social distancing) or interventions (vaccines, treatments, etc) on COVID-19 or societal outcomes. Eligible studies will then be assessed for components of social determinants. This will allow the research team to calculate the percentage of COVID-19 modelling studies that incorporated social determinants into their predicted outcomes of the pandemic (ie, studies that included social factors (numerator) and all eligible studies (denominator)). Data will only be extracted from models that included social determinants. The following studies will be excluded: (1) within-host biological studies, (2) non-human SARS-CoV-2 studies, (3) phylogenic/genetic studies, (4) environmental/ meteorological studies without health impact analysis on 


\section{Box 1 MEDLINE search strategy (4 August 2020)}

1. coronavirus/ or betacoronavirus/ or coronavirus infections/ (20740)

2. ("coronavirus"” or "coron?virinae*" or "novel coronavirus" or "novel corona virus" or "Severe Acute Respiratory Syndrome Coronavirus 2" or "coronavirus disease 2019" or "coronavirus pandemic?" or "coronavirus epidemic?" or "coronavirus outbreak?" or "corona virus pandemic?" or "corona virus epidemic?" or "corona virus outbreak?" or "corona virus disease 2019" or "new coronavirus" or "new corona virus" or "new coronaviruses" or "novel coronaviruses" or "2019 ncov" or "nCov 2019" or "SARS Coronavirus 2" or "2019-nCoV" or "2019nCoV" or "2019-CoV" or "nCoV2019" or "nCoV-2019" or "COVID-19" or "COVID-19" or "CORVID-19" or "CORVID19' or WN-CoV or WNCoV'" or "HCoV-19" or "HCoV19" or "CoV " or "2019 novel*" or "Ncov" or "nCov" or "nCOV" or "SARSCOV-2" or "SARSCoV-2" or "SARSCOV2" or "SARSCoV2" or "SARS-COV-2" or "SARSCov19" or "SARS-Cov19" or "SARSCOV-19" or "SARS-Cov-19" or "SARSr-Cov" or "Ncovor" or "Ncorona*" or "Ncorono*" or "NcovWuhan*" or "NcovHubei*" or "NcovChina*" or "NcovChinese*" or "Wuhan virus" or "novel CoV" or "CoV 2" or "CoV2" or "betacoron?vir*").mp. (54405)

3. ((corona* or corono*) adj1 (virus* or viral* or virinae $\left.\left.{ }^{\star}\right)\right)$.mp. (1032)

4. (((respiratory* adj2 (acute* or symptom* or disease* or illness ${ }^{\star}$ or infect* or condition*)) or "sea-food market*" or "seafood market*" or "food market" " or "foodmarket" or "wet market"” or "wetmarket*" or "wetmarket*") adj10 (Wuhan* or Hubei* or China* or Chinese* or Huanan*)).mp. (1479)

5. ((outbreak* or wildlife ${ }^{\star}$ or wild-life or pandemic ${ }^{\star}$ or epidemic ${ }^{*}$ or coronavirus or corona virus) adj3 (Wuhan* or Hubei* or China* or Chinese $^{\star}$ or Huanan*)).mp. (2228)

6. (anti-flu* or anti-influenza* or antiflu* or antinfluenza*).mp. (3379)

7. or/1-6 (60113) - (All Coronavirus / COVID-19 Terms)

8. Models, Theoretical/ (150928)

9. Models, Biological/ (338438)

10. Stochastic Processes/ or stochastic.mp. (44593)

11. (forecast* or model ${ }^{\star}$ or simulat*).af. (4238913)

12. or/8-11 (4250384) - (All Model Terms)

13. 7 and 12 (6971) - (All Coronavirus/COVID-19 Terms) AND (All Model Terms)

14. transmi*.ti,kf. (110754)

15. 7 and 14 (1788) - (Result Set to capture a non-COVID-19 paper)

16. 13 or $15(8442)$

17. (“32691014" or " 32689711 " or " 32171948 " or " 32687538 " or "32685697" or "32685143" or "32673577" or "32660125" or " 32546824 " or " 32372755 " or " 32246905 " or " 32325039 " or "32444481" or "32269020" or "32245814" or "32632012" or "22999128" or "22990082" or " 31422772 " or " 28577700 " or "24034486" or " 32691015 " or "32690354" or "32680824" or "32298421" or "22999128” or "22990082" or "22999132" or "31422772" or "28577700" or "24034486").ui. (26)

18. 13 and 17 (20)

19. limit 16 to $y \mathrm{r}=$ "2019 -Current" (4850)—(Final Result Set)

humans, (5) epidemiological/statistical analyses without a model, and (6) economic analyses without a model. An example search strategy can be found in box 1 .

\section{Review process}

After executing the search strategy, articles will be uploaded to Covidence software (v2619 b264f491) and duplicates will be removed. Titles and/or abstracts of the articles will be screened independently by two review members to identify studies that potentially meet the inclusion criteria outlined above. Conflicts will be resolved by a third independent reviewer. The full texts of these potentially eligible studies will then be retrieved and independently assessed for eligibility by two review team members. Any disagreements between them over the eligibility of particular studies will be resolved by a third reviewer. Data will then be extracted from the studies that incorporated social factors. A flow chart for this systematic review can be found in figure 1 .

\section{Data extraction and quality assessment}

Data will be extracted into Covidence by one reviewer and confirmed by a second reviewer. Disagreements will be resolved with a discussion between the two reviewers and if necessary, the final decision will be made by a third reviewer. If data related to the outcomes are missing, this will be reported. Authors will not be contacted to request missing data. The following data will be extracted from included studies: author, publication year, geography (country, province/state, city/town, region, low/middle/highincome country), setting (eg, general population, long-term care home, hospital, workplace, etc), population size, goals (predicting the pandemic, impact on other health conditions, impact on health services, impact of policy or interventions, economic analyses), type of model (mathematical, mechanistic, etc), social and economic factors, and approach to incorporating social and economic factors. An example extraction table containing the characteristics of included studies in the systematic review can be found in table 1 .

There is currently no standardised tool for assessing the quality of infectious disease modelling studies. We plan to adapt the principles of best practices for infectious disease modelling, laid out by Pitman et al into a tool for quality assessment of infectious disease models. ${ }^{24}$

\section{Evidence synthesis}

The extracted data will be used to create a narrative synthesis of the results. Based on our broad inclusion criteria, it is likely that the study designs and data will be too heterogeneous for meta-analyses to be appropriate. We expect to report on the number (per cent) of identified infectious disease models that incorporated social factors. We will report on the social factors considered, and indicate the number (per cent) of included models that considered each social factor. The narrative synthesis will describe how social factors were incorporated into the conceptualisation of the infectious disease models, estimate policy impact across social groups (if relevant) and describe which model types (eg, compartmental, network models) were used when social factors were considered. We will compare models in terms of the approaches used to incorporate social factors; identify trends and see if an increasing number of published models incorporated 


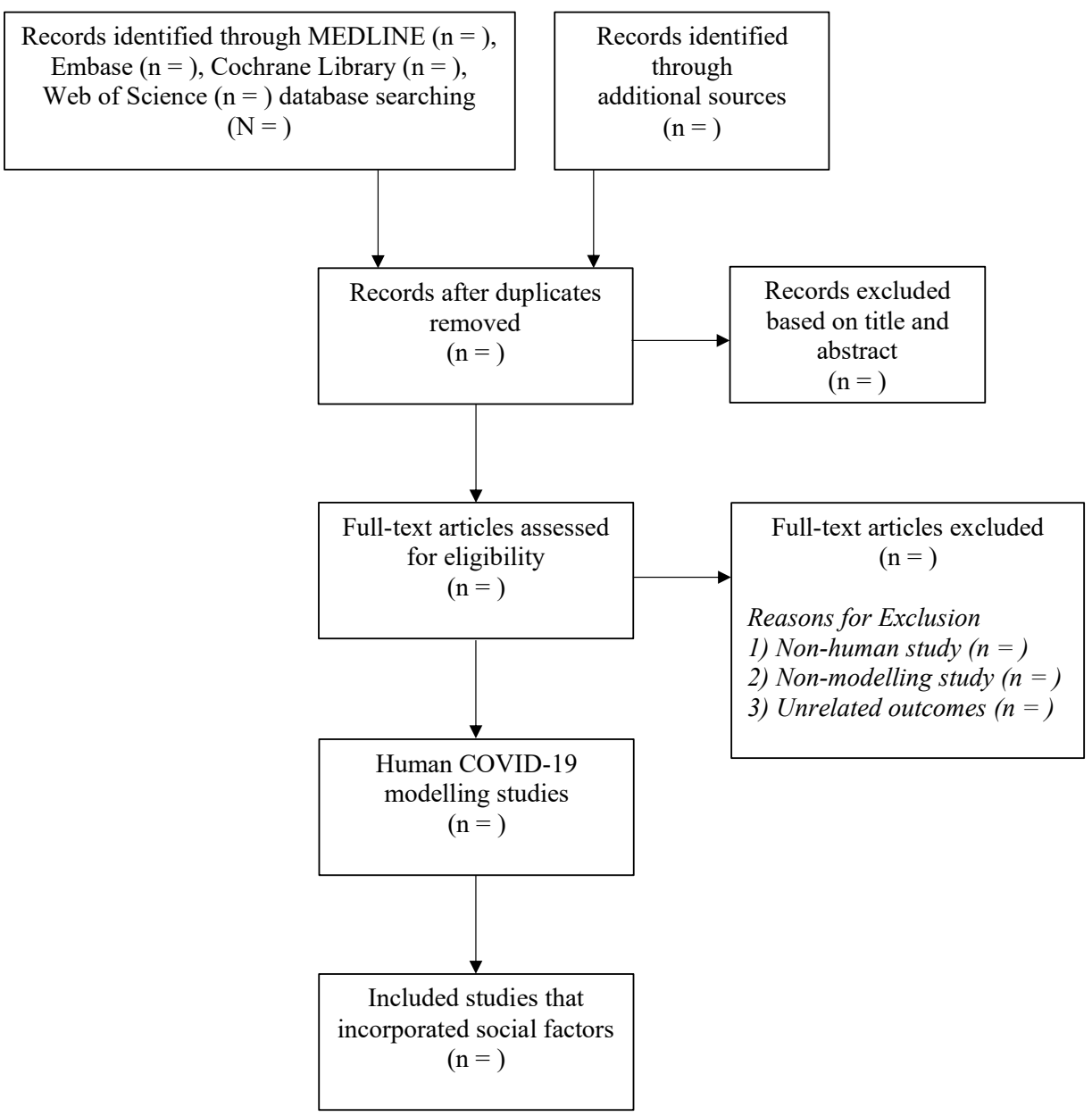

Figure 1 Flow diagram of selection criteria.

social factors over time; and based on our knowledge of published epidemiological estimates that quantify the association between social factors and COVID-19, ${ }^{25}$ compare incorporation of social factors in COVID-19 models to this evidence base.

\section{DISCUSSION}

To the best of our knowledge, this will be the first systematic review that aims to identify the frequency and approach used in COVID-19 models to incorporate the

Table 1 Dummy extraction table-characteristics of included studies

\begin{tabular}{|c|c|c|c|c|c|c|c|c|}
\hline Author(s) & $\begin{array}{l}\text { Month/ } \\
\text { year }\end{array}$ & Geography & Setting & $\mathbf{N}$ & Goal(s) & Model type & Social factors & $\begin{array}{l}\text { Approach to } \\
\text { social factors }\end{array}$ \\
\hline Smith et al & $\begin{array}{l}\text { March } \\
2020\end{array}$ & $\begin{array}{l}\text { Milan, } \\
\text { Italy } \\
\text { High income }\end{array}$ & Hospital & 400 & $\begin{array}{l}\text { Predicting the } \\
\text { pandemic } \\
\text { Case fatality }\end{array}$ & Compartmental & $\begin{array}{l}\text { Income } \\
\text { Employment }\end{array}$ & TBD \\
\hline Jones et al & $\begin{array}{l}\text { April } \\
2020\end{array}$ & $\begin{array}{l}\text { Wuhan, } \\
\text { China } \\
\text { High income }\end{array}$ & General & 2000 & $\begin{array}{l}\text { Impact of policy or } \\
\text { intervention } \\
\text { Lockdown }\end{array}$ & Network & Education & TBD \\
\hline
\end{tabular}

TBD, To be determined. 
social determinants of health to predict outcomes of the pandemic. Despite previous research and adequate knowledge of the disproportionate health burden experienced by marginalised groups, we hypothesise that the majority of COVID-19 models continue to ignore these important social factors. Without taking into account the influences of unequal levels of exposure, vulnerability and inequitable access to health services dictated by social factors, ${ }^{26}$ COVID-19 models may be providing an unclear illustration of the impact of COVID-19 and disease-control efforts. When health system decision-makers use average population-based models to implement large-scale health policies, they may be unintentionally exacerbating existing health inequities and leaving these marginalised groups further behind. Furthermore, failure to incorporate social factors may lead to missed opportunities to appraise targeted interventions to overcome inequities in the impact of COVID-19. Indeed, interventions targeted towards marginalised groups may prove more efficient for controlling the pandemic than interventions at the population level. Although our primary goal is to systematically document the scientific literature, our secondary goal with this review is to identify modellers that have incorporated social factors into the conceptualisation of their COVID-19 models, and detail the approach they used to do so. It is plausible that exclusion of social factors from COVID-19 models is not a result of neglect, but due to time constraints and limited computational power to handle increased model complexity. The modelling community is highly specialised and their skill sets are coveted by a range of policy decision-makers. Thus, modellers can have significant influence over policy decision-making processes. If modellers increase the awareness of the need to incorporate social factors into COVID-19 models, constraints on incorporating social factors into models can potentially be overcome through increased investments in computer infrastructure and strategic collaboration among modelling groups. It is our hope that this review will help to provide a preliminary roadmap for modellers to incorporate social factors into future COVID-19 models, and subsequently result in better health outcomes for the entire population.

The proposed systematic review will likely be limited by the rapidly evolving nature of the COVID-19 modelling literature. Given the time frame of the search strategy, we will likely not find many studies evaluating vaccination strategies. However, the findings on incorporating social factors into infectious disease models will still be relevant to modellers working on vaccine policy appraisal.

The strengths of the proposed review are that a systematic approach to evaluating modellers' incorporation of social factors into COVID-19 models can identify practical approaches to making better predictions and reduce inequities, quantify gaps in the literature, motivate enhancements in modelling best practices, and ultimately improve health for socially and economically vulnerable groups related to COVID-19 and other health conditions.

\section{Ethics and dissemination}

Ethical approval is not required as only secondary data will be collected. The results of this systematic review will be disseminated through peer-reviewed publication and conference proceedings.

Contributors AJ-B and SA secured the funding, designed the review, drafted the paper and approved the final version to be published. MM wrote the paper and approved the final version to be published.

Funding This project is funded by the Gordon and Betty Moore Foundation through grant GBMF9634 to Johns Hopkins University to support the work of the Society for Medical Decision Making COVID-19 Decision Modeling Initiative.

Competing interests None declared.

Patient and public involvement Patients and/or the public were not involved in the design, or conduct, or reporting, or dissemination plans of this research.

Patient consent for publication Not required.

Provenance and peer review Not commissioned; externally peer reviewed.

Open access This is an open access article distributed in accordance with the Creative Commons Attribution Non Commercial (CC BY-NC 4.0) license, which permits others to distribute, remix, adapt, build upon this work non-commercially, and license their derivative works on different terms, provided the original work is properly cited, appropriate credit is given, any changes made indicated, and the use is non-commercial. See: http://creativecommons.org/licenses/by-nc/4.0/.

ORCID iD

Marc S Moulin http://orcid.org/0000-0001-6654-4914

\section{REFERENCES}

1 Mein SA. COVID-19 and Health Disparities: the Reality of "the Great Equalizer". J Gen Intern Med 2020;35:2439-40.

2 Ali S, Asaria M, Stranges S. COVID-19 and inequality: are we all in this together? Can J Public Health 2020;111:415-6.

3 Williams DR, Cooper LA. COVID-19 and Health Equity-A New Kind of "Herd Immunity". JAMA 2020;323:2478-80.

4 Adhikari S, Pantaleo NP, Feldman JM, et al. Assessment of community-level disparities in coronavirus disease 2019 (COVID-19) infections and deaths in large US metropolitan areas. JAMA Netw Open 2020;3:e2016938

5 Caul S. Deaths involving COVID-19 by local area and socioeconomic deprivation: deaths occurring between 1 March and 31 July 2020. Statistical Bulletin 2020.

6 Hutchins SS, Fiscella K, Levine RS, et al. Protection of racial/ethnic minority populations during an influenza pandemic. Am J Public Health 2009;99 Suppl 2:S261-70.

7 Lessler J, Cummings DAT. Mechanistic models of infectious disease and their impact on public health. Am J Epidemiol 2016;183:415-22.

8 Star L, Moghadas SM. The role of mathematical modelling in public health planning and decision making. Purple Paper, National Collaborative Center for Infectious Diseases 2010.

9 Zhou L, Liu J-M, Dong X-P, et al. COVID-19 seeding time and doubling time model: an early epidemic risk assessment tool. Infect Dis Poverty 2020;9:1-9.

10 Tuite AR, Fisman DN, Reporting FDN. Reporting, epidemic growth, and reproduction numbers for the 2019 novel coronavirus (2019nCoV) epidemic. Ann Intern Med 2020;172:567-8.

11 Wang Y, Xu C, Yao S, et al. Forecasting the epidemiological trends of COVID-19 prevalence and mortality using the advanced $\alpha$-Sutte Indicator. Epidemiol Infect 2020;148:e236.

12 Ogden NH, Fazil A, Arino J. Artificial intelligence in public health: modelling scenarios of the epidemic of COVID-19 in Canada. Can Commun Dis Rep 2020;46:198-204.

13 Fisman DN, Greer AL, Tuite AR. Bidirectional impact of imperfect mask use on reproduction number of COVID-19: a next generation matrix approach. Infect Dis Model 2020;5:405-8. 
14 Tuite AR, Fisman DN, Greer AL. Mathematical modelling of COVID-19 transmission and mitigation strategies in the population of Ontario, Canada. CMAJ 2020;192:E497-505.

15 Wells CR, Fitzpatrick MC, Sah P, et al. Projecting the demand for ventilators at the peak of the COVID-19 outbreak in the USA. Lancet Infect Dis 2020;20:1123-5.

16 Roberts M, Russell LB, Paltiel AD, et al. Conceptualizing a model: a report of the ISPOR-SMDM modeling good research practices task Force-2. Med Decis Making 2012;32:678-89.

17 Briggs $\mathrm{AH}$, Weinstein MC, Fenwick EAL, et al. Model parameter estimation and uncertainty analysis: a report of the ISPOR-SMDM modeling good research practices Task force working Group-6. Med Decis Making 2012;32:722-32.

18 Marmot M. Social determinants of health inequalities. The Lancet 2005;365:1099-104.

19 Mauskopf J. Multivariable and structural uncertainty analyses for cost-effectiveness estimates: back to the future. Value Health 2019;22:570-4.
20 Jain R, Grabner M, Onukwugha E. Sensitivity analysis in costeffectiveness studies. Pharmacoeconomics 2011;29:297-314.

21 Sculpher M. Subgroups and heterogeneity in cost-effectiveness analysis. Pharmacoeconomics 2008;26:799-806.

22 Moher D, Shamseer L, Clarke M, et al. Preferred reporting items for systematic review and meta-analysis protocols (PRISMA-P) 2015 statement. Syst Rev 2015;4:1.

23 Booth A, Clarke M, Dooley G, et al. The nuts and bolts of Prospero: an international prospective register of systematic reviews. Syst Rev 2012;1:1-9.

24 Pitman R, Fisman D, Zaric GS, et al. Dynamic transmission modeling: a report of the ISPOR-SMDM modeling good research practices Task force working Group-5. Med Decis Making 2012;32:712-21.

25 Upshaw TL, Brown C, Smith R, et al. Social determinants of COVID-19 incidence and outcomes: a rapid review. PLoS One 2021;16:e0248336.

26 Moloughney BW. Social determinants of health: what can public health do to address inequities in infectious disease? Can Commun Dis Rep 2016;42:S1-14-S1-17. 